\title{
A non-autonomous second order boundary value problem on the half-line
}

\author{
Gregory S. Spradlin
}

\begin{abstract}
By variational arguments, the existence of a solution to a nonautonomous second-order boundary problem on the half-line is proven. The corresponding autonomous problem has no solution, revealing significant differences between the autonomous and the non-autonomous case.
\end{abstract}

Mathematics Subject Classification (2000). 34B40, 34C37.

Keywords. Heteroclinic, Non-autonomous equation, Bounded solution, Variational methods.

\section{Introduction}

Consider a nonnegative smooth scalar potential $V(x), x \in \mathbb{R}$, with $V(0)=$ $V(1)=0$ and $V>0$ on $(0,1) . a:[0, \infty) \rightarrow[0, \infty)$ and satisfies other assumptions which will be given later. We consider a boundary value problem of the form

$$
\begin{aligned}
& \ddot{x}(t)=a(t) V^{\prime}(x), \\
& x(0)=0, \quad x(t) \rightarrow 1 \text { as } t \rightarrow \infty .
\end{aligned}
$$

Boundary value problems for nonlinear second order ordinary differential equations on the half-line arise in physics, engineering, and biology applications. Of special interest is the existence of bounded solutions or solutions with a prescribed limit at infinity. The reader is referred to the first chapter of [1] for a survey on the subject. There is an extensive literature on problems like (1.1) on the whole line. The study of heteroclinic solutions is an important example of this kind of problem (see [2]).

If $a$ in (1.1) is positive and constant, a conservation of energy argument shows that (1.1)-(1.2) has no solutions. So there are significant differences between the autonomous case and the nonautonomous case. In [4], Coti Zelati and Rabinowitz studied non-autonomous $n$-dimensional systems and found heteroclinics that connect equilibria of different energy levels. Such solutions 
do not exist in the autonomous case, again by the conservation of energy principle. For higher-order equations, heteroclinic solutions between non-consecutive equilibria may exist (see the paper by Bonheure et al. [3]).

We prove the following:

Theorem 1.1. Let $V$ and a satisfy

$\left(V_{1}\right) V \in C^{1}(\mathbb{R})$ with $V(x) \geq 0$ for all $x \in \mathbb{R}, V>0$ on $(0,1), V(0)=V(1)=$ 0 .

$\left(V_{2}\right)$ There exist $\delta>0$ and $A_{1}, A_{2}>0$ with $A_{1} x^{2} \leq V(x) \leq A_{2} x^{2}$ for $0 \leq x \leq$ $\delta$.

$\left(A_{1}\right) a \in L^{\infty}([0, \infty))$, inf $a \geq 0, a(t) \rightarrow l>0$ as $t \rightarrow \infty$.

$\left(A_{2}\right) \lim _{t \rightarrow \infty}(l-a(t)) e^{2 \sqrt{2 l A_{1}} t}=\infty$.

Then (1.1)-(1.2) has a solution taking values on $[0,1]$.

Note that the assumptions preclude the case where $a$ is strictly decreasing everywhere. In this case, if $x$ satisfies (1.1)-(1.2), $E(t)=\frac{1}{2} \dot{x}(t)^{2}-a(t) V(x(t))$ is increasing and cannot converge to 0 as $t \rightarrow \infty$.

The theorem is stronger than that in a recent paper by Gavioli and Sanchez [5]. In their result, $V$ was assumed to be $C^{2}$, inf $a$ must be positive, $a$ must be of bounded variation, $a$ must be nondecreasing on $\left[t_{0}, \infty\right)$ for some $t_{0}>0$, and the coefficient of $t$ in condition $\left(A_{2}\right)$ is smaller. The arguments here are also more elementary than those of [5].

\section{Proof sketch}

Consider the autonomous equation

$$
\ddot{x}(t)=l V^{\prime}(x), \quad x(t) \rightarrow 0 \text { as } t \rightarrow-\infty, \quad x(t) \rightarrow 1 \text { as } t \rightarrow \infty .
$$

It is well-known that (2.1) has a solution $\omega$ that minimizes the functional

$$
I(u)=\int_{-\infty}^{\infty} \frac{1}{2} \dot{u}(t)^{2}+l V(u(t)) d t
$$

over the space $\left\{u \in W_{\text {loc }}^{1,2}(\mathbb{R}) \mid u(t) \rightarrow 0\right.$ as $t \rightarrow-\infty, u(t) \rightarrow 1$ as $\left.t \rightarrow \infty\right\}$. Let $\mathcal{B}=I(\omega)$. Define

$$
\begin{aligned}
X & =\left\{x \in W_{l o c}^{1,2}([0, \infty)) \mid x(0)=0, x(t) \rightarrow 1 \text { as } t \rightarrow \infty\right\}, \\
\mathcal{F}(x) & =\int_{0}^{\infty} \frac{1}{2} \dot{x}(t)^{2}+a(t) V(x(t)) d t .
\end{aligned}
$$

and $\mathcal{I}=\inf _{X} \mathcal{F}(x)$. We will show that $\mathcal{F}$ has a minimizer in $X$, which by standard arguments solves (1.1)-(1.2). The proof will follow from the following lemma and two propositions:

Lemma 2.1. If $x \in W_{l o c}^{1,2}([0, \infty)), \mathcal{F}(x)<\infty, 0 \leq x(t) \leq 1$ for all $t \geq 0$ and $x$ solves (1.1), then $x(t) \rightarrow 0$ or $x(t) \rightarrow 1$ as $t \rightarrow \infty$.

Proposition 2.2. If there exists a minimizing sequence $\left(x_{n}\right) \subset X$ for $\mathcal{F}$ with $0 \leq x_{n}(t) \leq 1$ for all $n \geq 1$ and $t \geq 0, x_{n} \rightarrow \bar{x}$ locally uniformly as $n \rightarrow \infty$, and $\sup \bar{x}<1$, and then $\mathcal{I} \geq \mathcal{B}$. 
Proposition 2.3. $\mathcal{I}<\mathcal{B}$.

Let $\left(x_{n}\right)$ be a minimizing sequence for $\mathcal{F}$. We may assume without loss of generality that $0 \leq x_{n}(t) \leq 1$ for all $n \geq 1$ and $t \geq 0$; otherwise, replace $x_{n}$ by $v_{n}=\max \left(0, \min \left(1, x_{n}\right)\right)$ with $\mathcal{F}\left(v_{n}\right) \leq \mathcal{F}\left(x_{n}\right) .\left(x_{n}\right)$ is bounded in $W^{1,2}([0, T])$ for all $T>0$, so along a subsequence, $\left(x_{n}\right)$ converges locally uniformly and weakly in $W^{1,2}([0, T])$ for all $T>0$ to $\bar{x}$. By standard arguments, $\bar{x}$ solves (1.1)-(1.2). By Fatou's Lemma, and the weak lower semicontinuity of $\int_{0}^{\infty} \dot{u}(t)^{2} d t, \mathcal{F}(\bar{x})<\infty$. By Lemma $2.1, \bar{x}(t) \rightarrow 0$ or $\bar{x}(t) \rightarrow 1$ as $t \rightarrow \infty$. Suppose $\bar{x}(t) \rightarrow 0$ as $t \rightarrow \infty$. Then if $\bar{x}(\hat{t})=1$ for any $\hat{t}>0$, we may redefine $\bar{x}(t)=1$ for all $t \geq \hat{t}$ and so $\bar{x}$ satisfies (1.1)-(1.2). Otherwise, $\sup \bar{x}<1$ and we may apply Proposition 2.2, and conclude that $\mathcal{I} \geq \mathcal{B}$. This contradicts Proposition 2.3. Therefore, $\bar{x}(t) \rightarrow 1$ as $t \rightarrow \infty$, and Theorem 1.1 is proven.

\section{Proofs of Lemmas and Propositions}

To prove Lemma 2.1, we need the following lemma:

Lemma 3.1. If $x \in X, 0 \leq x(t) \leq 1$ for all $t \geq 0$, and $x$ satisfies (1.1), then

$$
|\dot{x}(t)|<2+(\sup a)\left(\sup _{[0,1]}\left|V^{\prime}\right|\right)
$$

for all $t \geq 0$.

Proof. Suppose $\dot{x}\left(t^{\prime}\right) \geq b \equiv 2+(\sup a)\left(\sup _{[0,1]}\left|V^{\prime}\right|\right)$. Then for all $t \in\left[t^{\prime}, t^{\prime}+1\right]$,

$$
\begin{aligned}
\dot{x}(t) & =\dot{x}\left(t^{\prime}\right)+\int_{t^{\prime}}^{t} \ddot{x}(s) d s=\dot{x}\left(t^{\prime}\right)+\int_{t^{\prime}}^{t} a(s) V^{\prime}(x(s)) d s \\
& \geq b-(\sup a)\left(\sup _{[0,1]}\left|V^{\prime}\right|\right)=2
\end{aligned}
$$

and

$$
x\left(t^{\prime}+1\right)=x\left(t^{\prime}\right)+\int_{t^{\prime}}^{t^{\prime}+1} \dot{x}(t) d t \geq 0+2>1 .
$$

This is a contradiction. Similarly, $\dot{x}\left(t^{\prime}\right) \leq-b$ is impossible.

Proof of Lemma 2.1. Let $x \in W_{\text {loc }}^{1,2}([0, \infty)), \mathcal{F}(x)<\infty, x$ solves (1.1). Suppose $x(t) \nrightarrow 0$ and $x(t) \nrightarrow 1$ as $t \rightarrow \infty$. Then there exist $d \in(0,1 / 4)$ and a sequence $\left(t_{n}\right)$ with $t_{n} \rightarrow \infty$ as $n \rightarrow \infty$ and $d<x\left(t_{n}\right)<1-d$ for all $n$. Let $B=\min _{[d / 2,1-d / 2]} V>0$. Taking a subsequence of $\left(t_{n}\right)$ if necessary, assume

$$
t_{n+1}-t_{n}>b \equiv \frac{d / 2}{2+(\sup a)\left(\sup _{[0,1]}\left|V^{\prime}\right|\right)}
$$

for all $n$. By Lemma 3.1, for all $n \geq 1$ and $t \in\left[t_{n}, t_{n}+b\right]$,

$$
\begin{aligned}
x(t) & \leq x\left(t_{n}\right)+\int_{t_{n}}^{t} \dot{x}(s) d s \leq x\left(t_{n}\right)+\left(t-t_{n}\right)\left(2+(\sup a)\left(\sup _{[0,1]}\left|V^{\prime}\right|\right)\right) \\
& <(1-d)+d / 2=1-d / 2 .
\end{aligned}
$$


Similarly, for all $n \geq 1$ and $t \in\left[t_{n}, t_{n}+b\right], x(t)>d / 2$. So for all $n \geq 1$ and $t \in\left[t_{n}, t_{n}+b\right], d / 2<x(t)<1-d / 2$ and $V(x(t))>B$. Now

$$
\mathcal{F}(x) \geq \sum_{n=1}^{\infty} \int_{t_{n}}^{t_{n}+b} V(x(t)) d t \geq \sum_{n=1}^{\infty} \int_{t_{n}}^{t_{n}+b} B d t=\infty .
$$

This is a contradiction.

Proof of Proposition 2.2. Let $\left(x_{n}\right) \subset X$ be a minimizing sequence for $\mathcal{F}$ with $0 \leq x_{n}(t) \leq 1$ for all $n \geq 1, t \geq 0, x_{n} \rightarrow \bar{x}$ locally uniformly on $[0, \infty)$, and $\sup \bar{x}<1$. Let $\epsilon \in(0,1 / 4)$ with $\sup \bar{x}<1-\epsilon$. Define

$$
\mathcal{B}_{\epsilon}=\int_{\omega^{-1}(\epsilon)}^{\omega^{-1}(1-\epsilon)} \frac{1}{2} \dot{\omega}(t)^{2}+l V(\omega(t)) d t .
$$

Define $0<\alpha_{n}<\beta_{n}$ by

$$
\begin{aligned}
& \alpha_{n}=\max \left\{t \mid x_{n}(t)=\epsilon\right\}, \\
& \beta_{n}=\min \left\{t \mid t>\alpha_{n}, x_{n}(t)=1-\epsilon\right\} .
\end{aligned}
$$

Now,

$$
\int_{\alpha_{n}}^{\beta_{n}} \frac{1}{2} \dot{x}_{n}(t)^{2}+l V\left(x_{n}(t)\right) d t \geq \mathcal{B}_{\epsilon} .
$$

Otherwise, it would be possible to alter $\omega$ to $\tilde{\omega}$ with $\tilde{\omega} \in W_{l o c}^{1,2}(\mathbb{R}), \tilde{\omega}(t) \rightarrow 0$ as $t \rightarrow-\infty, \tilde{\omega}(t) \rightarrow 1$ as $t \rightarrow \infty$, and $I(\tilde{\omega})<\mathcal{B}$, contradicting the optimality of $\omega$. $\tilde{\omega}$ is constructed by replacing $\left.\omega\right|_{\left[\omega^{-1}(\epsilon), \omega^{-1}(1-\epsilon)\right]}$ with $\left.x_{n}\right|_{\left[\alpha_{n}, \beta_{n}\right]}$ :

$$
\tilde{\omega}(t)= \begin{cases}\omega(t) ; & t \leq \omega^{-1}(\epsilon) \\ x_{n}\left(t-\omega^{-1}(\epsilon)+\alpha_{n}\right) ; & \omega^{-1}(\epsilon) \leq t \leq \omega^{-1}(\epsilon)+\beta_{n}-\alpha_{n} \\ \omega\left(\omega^{-1}(1-\epsilon)+t-\omega^{-1}(\epsilon)-\left(\beta_{n}-\alpha_{n}\right)\right) ; & t \geq \omega^{-1}(\epsilon)+\beta_{n}-\alpha_{n} .\end{cases}
$$

Since $x_{n} \rightarrow \bar{x}$ locally uniformly as $n \rightarrow \infty$ and $\sup \bar{x}(t)<1-\epsilon, \beta_{n} \rightarrow \infty$ as $n \rightarrow \infty . x_{n}(t) \in[\epsilon, 1-\epsilon]$ for $\alpha_{n} \leq t \leq \beta_{n}$ so $V\left(x_{n}(t)\right) \geq b_{\epsilon} \equiv \min _{[\epsilon, 1-\epsilon]} V>0$ for $\alpha_{n} \leq t \leq \beta_{n}$.

Let $\hat{t}>0$ be large enough that $a \geq l / 2$ on $[\hat{t}, \infty)$. This is possible by $\left(A_{2}\right)$. For large $n, \alpha_{n} \geq \hat{t}$; otherwise, along a subsequence,

$$
\begin{aligned}
\mathcal{F}\left(x_{n}\right) & \geq \int_{\alpha_{n}}^{\beta_{n}} a(t) V\left(x_{n}(t)\right) d t \geq \int_{\hat{t}}^{\beta_{n}} a(t) V\left(x_{n}(t)\right) d t \\
& \geq(l / 2) b_{\epsilon}\left(\beta_{n}-\hat{t}\right) \rightarrow \infty
\end{aligned}
$$

as $n \rightarrow \infty$. $\left(\beta_{n}-\alpha_{n}\right)$ is bounded, since

$$
\mathcal{F}\left(x_{n}\right) \geq \int_{\alpha_{n}}^{\beta_{n}} a(t) V\left(x_{n}(t)\right) d t \geq(l / 2) b_{\epsilon}\left(\beta_{n}-\alpha_{n}\right) .
$$


Therefore $\alpha_{n} \rightarrow \infty$ as $n \rightarrow \infty$. Let $n$ be large enough so that $a \geq l-\epsilon$ on $\left[\alpha_{n}, \infty\right)$. For such $n$,

$$
\begin{aligned}
\mathcal{F}\left(x_{n}\right) & \geq \int_{\alpha_{n}}^{\beta_{n}} \frac{1}{2} \dot{x}_{n}(t)^{2}+(l-\epsilon) V\left(x_{n}(t)\right) d t \\
& \geq \frac{l-\epsilon}{l} \int_{\alpha_{n}}^{\beta_{n}} \frac{1}{2} \dot{x}_{n}(t)^{2}+l V\left(x_{n}(t)\right) d t \geq\left(1-\frac{\epsilon}{l}\right) \mathcal{B}_{\epsilon} .
\end{aligned}
$$

$\mathcal{B}_{\epsilon} \rightarrow \mathcal{B}$ as $\epsilon \rightarrow 0$, so $\mathcal{I}=\lim _{n \rightarrow \infty} \mathcal{F}\left(x_{n}\right) \geq \mathcal{B}$.

Before proving Proposition 2.3, we need the following lemma:

Lemma 3.2. There exists $C>0$ such that $\omega(t) \leq C e^{\sqrt{2 A_{1}} t}$ for all $t \in \mathbb{R}$.

Proof. $\omega$ solves $\ddot{\omega}=l V^{\prime}(\omega(t))$. Multiplying both sides by $\dot{\omega}$ and integrating yields $\frac{1}{2} \dot{\omega}(t)^{2}-l V(\omega(t))=c$, where $c$ is a constant. $\dot{\omega}(t) \rightarrow 0$ and $V(\omega(t)) \rightarrow 0$ as $t \rightarrow-\infty$, so $c=0$. Taking square roots yields $\dot{\omega}(t)=\sqrt{2 l V(\omega(t))}$. Let $\tilde{t} \in \mathbb{R}$ with $\omega(t) \leq \delta$ for $t \leq \tilde{t}$. For $t \leq \tilde{t}$,

$$
\begin{gathered}
\dot{\omega}(t)=\sqrt{2 l V(\omega(t))} \geq \sqrt{2 l A_{1}} \omega(t), \\
e^{-\sqrt{2 l A_{1}} t} \dot{\omega}(t)-\sqrt{2 l A_{1}} e^{-\sqrt{2 l A_{1}} t} \omega(t) \geq 0, \quad \frac{d}{d t}\left(e^{-\sqrt{2 l A_{1}} t} \omega(t)\right) \geq 0, \\
e^{-\sqrt{2 l A_{1}} t} \omega(t) \leq e^{-\sqrt{2 l A_{1}} \tilde{t}} \omega(\tilde{t}), \quad \omega(t) \leq e^{-\sqrt{2 l A_{1}} \tilde{t}} \omega(\tilde{t}) e^{\sqrt{2 l A_{1}} t} .
\end{gathered}
$$

Using a larger constant than $e^{-\sqrt{2 l A_{1}} \tilde{t}} \omega(\tilde{t})$, the inequality is extended to all $t \in \mathbb{R}$.

Proof of Proposition 2.3. Let $A_{1}$ and $A_{2}$ be as in $\left(V_{2}\right)$ and $C$ as in Lemma 3.2. Let $t_{0}>1$ and be large enough that $a \leq l$ on $\left[t_{0}, \infty\right)$. Let

$$
K=\frac{2 C^{2}\left(1+A_{2}(l+\sup a)\right) e^{2 t_{0} \sqrt{2 l A_{1}}}}{\sqrt{l A_{1}} \int_{-1}^{0} V(\omega(t)) d t} .
$$

Let $T>t_{0}+1$ be large enough that $(l-a(t)) e^{2 \sqrt{2 l A_{1}} t} \geq K$ for $t \geq T-1$ and $\omega(t) \leq \delta$ for $t \leq t_{0}-T$. Let $x_{T}$ be $\omega$ translated to the right by $T$ and truncated at $t=0$ so $x_{t} \in X$ :

$$
x_{T}(t)= \begin{cases}t \omega(t-T) ; & 0 \leq t \leq 1 \\ \omega(t-T) ; & t \geq 1\end{cases}
$$

We will show that $\mathcal{F}\left(x_{T}\right)<\mathcal{B}$, proving $\mathcal{I}<\mathcal{B}$.

$$
\begin{aligned}
\mathcal{F}\left(x_{T}\right)-\mathcal{B}= & \int_{0}^{\infty} \frac{1}{2} \dot{x}_{T}(t)^{2}+a(t) V\left(x_{T}(t)\right) d t \\
& -\int_{-\infty}^{\infty} \frac{1}{2} \dot{\omega}(t-T)^{2}+l V(\omega(t-T)) d t \\
= & \int_{0}^{1} \frac{1}{2}(t \dot{\omega}(t-T)+\omega(t-T))^{2}+a(t) V(t \omega(t-T)) d t
\end{aligned}
$$




$$
\begin{aligned}
& +\int_{1}^{t_{0}} \frac{1}{2} \dot{\omega}(t-T)^{2}+a(t) V(\omega(t-T)) d t \\
& +\int_{t_{0}}^{T-1} \frac{1}{2} \dot{\omega}(t-T)^{2}+a(t) V(\omega(t-T)) d t \\
& +\int_{T-1}^{T} \frac{1}{2} \dot{\omega}(t-T)^{2}+a(t) V(\omega(t-T)) d t \\
& +\int_{T}^{\infty} \frac{1}{2} \dot{\omega}(t-T)^{2}+a(t) V(\omega(t-T)) d t \\
& -\int_{-\infty}^{t_{0}} \frac{1}{2} \dot{\omega}(t-T)^{2}+l V(\omega(t-T)) d t \\
& -\int_{t_{0}}^{T-1} \frac{1}{2} \dot{\omega}(t-T)^{2}+l V(\omega(t-T)) d t \\
& -\int_{T-1}^{T} \frac{1}{2} \dot{\omega}(t-T)^{2}+l V(\omega(t-T)) d t \\
& -\int_{T}^{\infty} \frac{1}{2} \dot{\omega}(t-T)^{2}+l V(\omega(t-T)) d t \\
& \leq \int_{0}^{1} \dot{\omega}(t-T)^{2}+\omega(t-T)^{2}+(\sup a) A_{2} C^{2} e^{2 \sqrt{2 l A_{1}}(t-T)} d t \\
& +\int_{1}^{t_{0}} \frac{1}{2} \dot{\omega}(t-T)^{2}+(\sup a) A_{2} C^{2} e^{2 \sqrt{2 l A_{1}}(t-T)} d t \\
& +\int_{T-1}^{T}(a(t)-l) V(\omega(t-T)) d t \\
& \leq \int_{-T}^{1-T} 2 l V(\omega(t))+\omega(t)^{2}+(\sup a) A_{2} C^{2} e^{2 \sqrt{2 l A_{1}} t} d t \\
& +\int_{1-T}^{t_{0}-T} l V(\omega(t))+(\sup a) A_{2} C^{2} e^{2 \sqrt{2 l A_{1}} t} d t \\
& -K e^{-2 \sqrt{2 l A_{1}}(T-1)} \int_{-1}^{0} V(\omega(t)) d t \\
& \leq C^{2}\left(2 l A_{2}+1+A_{2}(\sup a)\right) \int_{-\infty}^{1-T} e^{2 \sqrt{2 l A_{1}} t} d t \\
& +A_{2} C^{2}(l+(\sup a)) \int_{-\infty}^{t_{0}-T} e^{2 \sqrt{2 l A_{1}} t} d t \\
& -K e^{-2 \sqrt{2 l A_{1}}(T-1)} \int_{-1}^{0} V(\omega(t)) d t
\end{aligned}
$$




$$
\begin{aligned}
\leq & C^{2}\left(1+2 A_{2}(l+(\sup a))\right) \int_{-\infty}^{t_{0}-T} e^{2 \sqrt{2 l A_{1}} t} d t \\
& -K e^{-2 \sqrt{2 l A_{1}}(T-1)} \int_{-1}^{0} V(\omega(t)) d t \\
= & \left(\frac{C^{2}\left(1+2 A_{2}(l+(\sup a))\right)}{2 \sqrt{2 l A_{1}}} e^{2 \sqrt{2 l A_{1}} t_{0}}-K e^{2 \sqrt{2 l A_{1}}} \int_{-1}^{0} V(\omega(t)) d t\right) \\
& \times e^{-2 \sqrt{2 l A_{1}} T}<0
\end{aligned}
$$

Proposition 2.3 is proven.

\section{References}

[1] Aggarwal, R.P., O'Regan, D.: Infinite Interval Problems for Differential, Difference, and Integtral Equations. Kluwer, Dordrecht (2001)

[2] Bonheure, D., Sanchez, L.: Heteroclinic orbits for some classes of second and fourth order differential equations. In: Canãda, A., Drabek, P., Fonda, A. (eds.) Handbook of Differential Equations III. Elsevier, North Holland (2006)

[3] Bonheure, D., Sanchez, L., Tarallo, M., Terracini, S.: Heteroclinic connections between nonconsecutive equilibria of a fourth order differential equation. Calc. Var. Partial Differ. Equ. 17, 341-356 (2001)

[4] Coti Zelati, V., Rabinowitz, P.H.: Heteroclinic solutions between stationary points at different energy levels. Top. Math. Nonlinear Anal. 17, 1-21 (2001)

[5] Gavioli, A., Sanchez, L.: On a class of bounded trajectories for some non-autonomous systems. Math. Nachr. 281(11), 1557-1565 (2008)

\section{G. S. Spradlin}

Department of Mathematics,

Embry-Riddle University,

Daytona Beach, FL 32114-3900,

USA

e-mail: spradlig@erau.edu

Received: 22 June 2009.

Accepted: 25 March 2010. 